\title{
$\$$ Research Square \\ CT-Based Quantitative Assessment of Coronavirus Disease 2019 Using a Deep Learning-Based Segmentation System: A Longitudinal Study
}

\section{Jianneng Li}

Guangdong Second Provincial General Hospital

\section{Wei Zuo}

Guangdong Second Provincial General Hospital

Qiang Lei

Guangdong Second Provincial General Hospital

\section{Guihua Jiang}

Guangdong Second Provincial General Hospital

Jianhao yan ( $\nabla$ yanjianhao@163.com )

Guangdong Second Provincial General Hospital https://orcid.org/0000-0002-5670-1207

\section{Long Li}

Guangdong Provincial Corps Hospital of Chinese People's Armed Police Forces

\section{Research}

Keywords: Coronavirus disease 2019, Pneumonia, Quantitative assessment, Deep learning, Computed tomography

Posted Date: May 12th, 2021

DOl: https://doi.org/10.21203/rs.3.rs-504170/v1

License: (9) This work is licensed under a Creative Commons Attribution 4.0 International License. Read Full License 


\section{Abstract}

\section{Background}

Coronavirus disease 2019 (COVID-19) is a global catastrophic disease that has severely affected more than 185 countries. The key steps in fighting against COVID-19 involve early detection and tracking of the treatment effects. A large number of studies highlighted computed tomography (CT) as a reliable method for early diagnosis and follow-up monitoring of the disease. However, there are limited data on quantitative analysis of the follow-up images. In this study, we used a deep learning model using a neural network with high accuracy in automatic segmentation and quantification to analyze the infected lesions on chest CT images.

Methods

We used a deep learning model using a neural network with high accuracy in automatic segmentation and quantification to analyze the infected lesions on chest CT images. A total of 14 patients (mean age, $53 \pm 14$ years; age range, $23-74$ years; $42.9 \%$ men and $57.1 \%$ women) with confirmed mild-type COVID-19 from January 1 to May 7, 2020, were retrospectively reviewed. Initial and follow-up original CT images were collected, and CT quantitative parameters, including percentage of infection (POI) and density variation of pneumonia, were determined.

Results

The median initial POI was 3.4\% (interquartile range, IQR $0.5 \%-8.4 \%$ ) for the whole lung, $0.8 \%$ (IQR $0.2 \%$ $6.7 \%$ ) for the left lung, and $5.8 \%$ (IQR $0.5 \%-9.7 \%$ ) for the right lung. The infection was more serious in the right than in the left lung. The infected region mainly involved bilateral lower lobes, more pronounced on the right side. Quantitative CT showed that POI significantly decreased throughout the follow-up period in all 14 patients $(p<0.001)$. Among them, $50 \%$ of the patients had a more significant decrease in POI $(51.3 \%)$ after a negative nucleic acid test. Moreover, there was a significant decrease in the CT number range of ground-glass opacities (GGO) and consolidation $(p<0.001)$.

Conclusions

This study demonstrated the quantitative analysis of follow-up CT scans plays an important role in the monitoring of COVID-19 treatment, which could help in treatment planning and standardizing the assessment for discharge.

\section{Background}

The outbreak of a novel coronavirus pneumonia has rapidly spread from Wuhan, China to all major global regions since December 2019 [1, 2]. This disease was named Coronavirus disease 2019 (COVID19) by the World Health Organization (WHO) [3]. To date (March 24, 2021), there have been 110.7 million confirmed cases and over 2.4 million deaths globally since the start of the pandemic [4]. Based on 
epidemiological characteristics, clinical manifestations, chest imaging, and laboratory examination results are the main screening tools for identifying COVID-19 infection. The disease is typically confirmed by reverse-transcription polymerase chain reaction (RT-PCR) $[5,6]$. However, a high false-negative rate of RT-PCR has been reported in the early stage of COVID-19 infection, which may lead to missed diagnosis and increase the risk of further disease spread $[5,7]$. In addition, considering the season of respiratory diseases and similar symptoms that need to be quickly identified, the time required to do the RT-PCR test can delay appropriate patient isolation and treatment. Chest computed tomography (CT) has been used for screening of COVID-19 due to high sensitivity, thereby serving as an important complement to the RTPCR testing [5]. During the treatment of patients with confirmed COVID-19, CT examination is considered a supplement to clinical evaluation and laboratory tests [14]. Hence, the CT findings play a critical role in constraining the viral transmission and fighting against COVID-19.

Due to rapid progress of the disease, follow-up CT scans every 3-5 days have been recommended by clinicians to evaluate the response to treatment. Moreover, serial chest CT imaging performed at different time points is also effective in estimating the evolution of the disease from initial diagnosis to discharge. It has been reported that follow-up CT examination can evaluate the progression during the early stage of hospitalized COVID-19 patients [8, 9]. However, these CT examinations undoubtedly put tremendous work pressure on radiologists. Moreover, subtle changes in follow-up CT scans are often ignored due to lack of computerized tools to accurately quantify the infected regions of the lungs and their longitudinal changes. Jin et al. [10] proposed an artificial intelligence (AI) system based on CT images for fast COVID19 diagnosis. Al, a relatively mature technology in the field of medical imaging [11, 12], has actively contributed to the fight against COVID-19 [13]. A previous study showed that not only Al using deep learning (DL) in chest CT images could quickly identify and automatically contour infected regions, but it could also accurately estimate their shape, volume, and percentage of infection (POI), as well as accurately locate the infected lung lobe and bronchopulmonary segment [13]. Thus, the use of Al to support screening and clinical medical decisions is a useful tool in fighting against COVID-19.

Most of the current research has focused on the screening and differential diagnosis of COVID-19, whereas studies of COVID-19 follow-up remain limited. The use of DL-based quantitative analysis of chest CT can not only help radiologists in assessment of a rising number of examinations but also help clinicians to acquire results much faster, in order to rapidly apply appropriate treatment. In this study, we used a DL-based segmentation system for quantitative assessment of the follow-up CT images of the whole lung and lobes affected by COVID-19 to measure the severity of the disease and the distribution of infection within the lung, and to provide the tracking of longitudinal changes during the course of treatment. In addition, this study also applied DL-based quantitative analysis to chest CT scans to standardize the discharge criteria of hospitalized COVID-19 patients with two consecutive negative nucleic acid tests.

\section{Materials And Methods}


This study was approved by our local institutional review board (IRB), and necessity for further consent was waived because the study was retrospective research that would pose no potential risk to patients and would not adversely affect the patients' right of welfare. From the hospital medical record system, we included 37 patients with COVID-19 confirmed by RT-PCR (mean age, 45 \pm 14 years; age range, 12-74 years; $54.1 \%$ men and $45.9 \%$ women), who were admitted to the hospital between January 1 and May 7 , 2020. COVID-19 manifested with non-respiratory symptoms as well as respiratory symptoms, which were nonspecific and of variable severity, ranging from minimal $(n=5)$ and mild $(n=31)$ to severe and critical disease $(n=1)$. The inclusion criteria were as follows: 1$)$ a positive result of RT-PCR test confirmed by the Centers for Disease Control (CDC), or positive antibodies against new coronavirus nucleic acid; 2 ) mild disease (non-severe and non-critical patients); 3 ) age $\geq 18$ years; 4 ) availability of complete laboratory examinations and CT data; 5) successfully completed high-resolution thin-layer CT examinations during the study period, with a layer thickness $\leq 1 \mathrm{~mm}$; 6) lung infection in CT images; and 7) more than two follow-up records of CT. The exclusion criteria were as follows: 1 ) minimal disease (positive nucleic acid test, but no infection in chest CT images) and severe or critical disease [14]; 2) contrast chest CT examination; 3) CT examination with slice thickness $>1 \mathrm{~mm}$; 4) large motion artifacts or pre-existing obvious signs of other lung disease on CT images; and 5) only one follow-up CT record. The following discharge criteria were followed: 1 ) normal body temperature for more than 3 days; 2) obviously improved respiratory symptoms; 3) significant improvement of infected lesions on chest CT; 4) two consecutive respiratory samples tested negative for SARS-CoV-2 nucleic acid; and 5) the sample collection times were at least 24 hours apart.

\section{Clinical data}

Electronic medical records of the patients were reviewed. Specific clinical information, including fever, cough, sore throat, fatigue, and muscle soreness, was collected from the medical records of all patients. Fever was defined as a body temperature $>37.3^{\circ} \mathrm{C}$. To confirm COVID-19, nasopharyngeal and oropharyngeal swabs received at a time interval of 24 hours were used for RT-PCR testing in each patient. The RT-PCR test kits used in this study were manufactured by Da An Gene Co. (Guangzhou, China). In addition, the RT-PCR test was considered negative when two consecutive results were negative. Mild type of COVID-19 was defined as patients with slight clinical symptoms and no signs of pneumonia on CT imaging. Mild-type COVID-19 was defined as symptoms of fever and/or signs of respiratory infection, and signs of pneumonia on CT imaging. All of the patients underwent follow-up CT scans after antiviral drug treatment. Patient demographic characteristics, epidemiological characteristics, clinical manifestations, and laboratory results were collected.

\section{CT image data acquisition}

A high-resolution CT scan was performed in all patients using multi-slice spiral CT scanners (Philips Brilliance-16, Philips iCT-256, Cleveland, USA). The scanning followed the common chest protocol with standard dose, where all patients were placed in a supine position, with arms raised, and held their breath 
during acquisition. The scanning range included whole lung volume. The following scanning parameters were applied: tube voltage, $120 \mathrm{kV}$; automatic tube current, $180 \mathrm{~mA}$; pitch, 1.06; rotation time, $0.5 \mathrm{~s}$; slice thickness, $0.8 \mathrm{~mm}$; slice increment, $0.4 \mathrm{~mm}$; image matrix, $1024 \times 1024$; and window width/level 1500/-600 Hounsfield units (HU) for lung window setting.

\section{DL-based network and quantitative assessment}

We used a DL-based network called VB-Net (based on United Imaging Intelligence, Shanghai, China), which is a modified three-dimensional convolutional neural network $[15,16]$. This DL-based VB-Net system was developed to automatically segment and quantify the infected regions in CT scans of COVID19 patients. After original CT images had been acquired, these DICOM data were fed to the VB-Net system. Segmentation was an essential step in VB-Net for image processing and analysis. It delineated the regions of interest (ROIs) in CT images, including whole lungs, lung lobes, and bronchopulmonary segments, as well as infected regions or lesions. After segmentation, various metrics were computed to quantify the degree of COVID-19 infection. The results of quantitative evaluation showed high accuracy (up to $91.6 \%$ ) for automatic segmentation of the infected region and determination of percentage of infection (POI) metrics (15). In this study, we used VB-Net to quantitatively evaluate the lung infection regions of the initial and follow-up CT images. The volumes and POls of infection were quantified for the whole lung and for each lobe. The histogram of Hounsfield units (HU) within the infected region was also calculated to evaluate the density variation of pneumonia detected by $\mathrm{DL}$, including ground-glass opacities (GGOs), consolidation components, and fibrosis inside the infected lesions. The ranges of CT numbers for GGOs, consolidation components, and fibrosis were set at <-300 HU, $-300 \mathrm{HU}$ to $49 \mathrm{HU}$, and $\geq 50 \mathrm{HU}$, respectively. The quantification provided a basis for tracking longitudinal changes of COVID-19 during the course of treatment from a CT perspective.

\section{Statistical analysis}

Statistical analyses were performed using IBM SPSS Statistics (version 19.0, SPSS Inc., United States). The measured data are presented as mean \pm standard deviation (mean \pm SD) or median and interquartile range (IQR, the first to the third quartile) when the continuous data did not follow a normal distribution. The differences between the baseline and follow-up measurements were evaluated by Friedman test, which is a nonparametric test for multiple paired samples. $P<0.05$ was considered statistically significant.

\section{Results}

\section{Epidemiology and clinical characteristics}

Thirty-seven patients with COVID-19 were initially enrolled. Twenty-three patients (62.3\%) were excluded because they did not match the inclusion criteria. Finally, a total of 14 patients (mean age, $53 \pm 14$ years; 
age range, $23-74$ years; $42.9 \%$ men and $57.1 \%$ women) with mild-type COVID-19 were included. All 14 patients had a history of exposure to COVID-19 (10 of 14 cases lived in Hubei province, China; 4 of 14 cases were exposed to an infected patient). The initial symptoms included fever $\left(37.7 \pm 0.2^{\circ} \mathrm{C}\right.$; in $12 / 14$, $85.7 \%$ ), cough (in $5 / 14,35.7 \%$ ), and sore throat (in $2 / 14,14.3 \%$ ). Major abnormal laboratory findings included a reduced lymphocyte count $\left(0.9 \pm 0.1 \times 10^{9} / \mathrm{L}\right.$; in $\left.11 / 14,78.6 \%\right)$, reduced leukocyte count (3.2 $\pm 0.4 \times 10^{9} / \mathrm{L}$; in 5/14, 35.7\%), reduced $\mathrm{CD}^{+} \mathrm{T}$ lymphocyte count $(512.7 \pm 131.7 / \mu \mathrm{L}$; in $12 / 14,85.7 \%)$, reduced CD $4^{+} \mathrm{T}$ lymphocyte count $(307 \pm 78.2 / \mu \mathrm{L} ;$ in $12 / 14,85.7 \%)$, reduced $\mathrm{CD} 8^{+} \mathrm{T}$ lymphocyte count $(142 \pm 57.2 / \mu \mathrm{L}$; in $8 / 14,57.1 \%)$, and elevated C-reactive protein $(27.8 \pm 18.8 \mathrm{mg} / \mathrm{L}$; in $10 / 14,71.4 \%)$. Six patients $(42.9 \%)$ had comorbidities (hyperuricemia, hypertension, chronic hepatitis $\mathrm{B}$, and chronic obstructive pulmonary disease) (Table 1 ). The average hospitalization lasted $21 \pm 9$ days. These inpatients had negative nucleic acid results via RT-PCR test after an average of $12 \pm 7$ days; however, the RT-PCR test of six inpatients again turned positive ("re-positive") during the follow-up.

\section{Initial and follow-up CT findings}

Fourteen patients underwent at least two chest CT scans, as per inclusion criteria. The follow-up CT scans every $3-5$ days were recommended by clinicians to evaluate the therapeutic response in the early stage of COVID-19. In our cases, the interval of repeated CT scanning was 3-17 days. All initial CT scans showed signs of pneumonia after achieving positive RT-PCR results. The initial CT images of all 14 patients showed abnormal pulmonary findings with irregular patches of pure GGOs (17.4\%), the mixed pattern of GGOs and consolidation components (47.8\%), and consolidation components with fibrosis (34.8\%). There were three cases in which only one lung lobe was involved. In contrast, in 11 cases, multiple lobes were bilaterally affected, including 10 cases in the left upper lobe, 11 cases in the left lower lobe, 11 cases in the right upper lobe, 8 cases in the right middle lobe, and 12 cases in the right lower lobe. Apparently, the lower lobes of both lungs were often affected. A gradual decrease in GGO regions and increased consolidation components with reticulations and stripes were the main findings at the follow-up CT scans. Finally, the latest CT scans showed complete absorption of GGO and reduction of consolidation components after antiviral and supportive treatment.

\section{Quantitative results of the initial and follow-up CT images}

Some of the VB-Net system's predictions proposed in this paper are shown in Figure 1. Quantitative results from the initial and follow-up CT images obtained using VB-Net are presented in Figures 2 and 3 and Table 2. Table 2 shows that the median POI in the whole lung, left lung, and right lung was $3.4 \%$ (IQR $0.5 \%-8.4 \%), 0.8 \%$ (IQR $0.2 \%-6.7 \%$ ), and $5.8 \%$ (IQR $0.5 \%-9.7 \%$ ), respectively. This indicates that the infection was more serious in the right than in the left lung, and that the infected region was mainly distributed in the lower lobes of both lungs, especially in the right lower lobe with a median POI of $7.5 \%$ (IQR $0.7 \%-19.4 \%$ ). In addition, the median POI of the infected region was $3.1 \%$ (IQR $0.5 \%-7.0 \%$ ), $0.4 \%$ (IQR $0.0 \%-1.3 \%$ ), and $0.0 \%$ (IQR $0.0 \%-0.1 \%$ ) for the CT number ranges of <-300 HU, $-300 \mathrm{HU}$ to $49 \mathrm{HU}$, 
and $\geq 50 \mathrm{HU}$, respectively, indicating that the GGOs were the most extensive component, while consolidation and fibrosis were formed in some lesions. The 3D display and POI of the lesions are also shown in Figure 3.

In 14 patients, the quantitative analysis revealed significantly decreased POIs $(p<0.001)$ at the follow-up compared with the baseline (Table 2), especially after being tested negative on RT-PCR test. This showed that after antiviral and supportive treatment, the infection area gradually decreased, and the lesions of the whole lung, left lung, and right lung in those patients improved accordingly (Table 2). Three to seven days after receiving antiviral and supportive treatment, quantitative results of the first follow-up revealed that most lesions improved and were absorbed. However, two patients showed obviously disease progression at the first follow-up, demonstrating an increased infected area and consolidation components in bilateral multiple lobes. In addition, six patients showed an increase in the consolidation components compared with the initial scan. Three patients showed a slight disease progression at the second and third followups. All patients showed improvement at the last follow-up (Figure 2). By the last follow-up, the infected lesions had been almost completely absorbed in nine patients, and the POls were lower than $3 \%$ in other four patients. The infected lesions in these patients contained less than $2 \%$ of consolidation components and less fibrosis compared with the initial CT scans. However, one patient showed different findings. Namely, because the infection area was large, involving $40.1 \%$ of the whole lung, and more consolidation components and fibrosis formed locally inside the lesions, the absorption time of the lesion was longer than in other patients. However, the POls of all 14 patients showed that the CT number range of GGO and consolidation significantly decreased $(p<0.001)$. These follow-up results suggested that clinically relevant supportive treatment can be reflected by changes in POls.

In addition, the RT-PCR test results of six inpatients again turned positive ("re-positive") during the followup period. However, the quantitative follow-up CT showed that there was no increase in the infected lesions. Our DL-determined discharge standard of the follow-up CT showed that the POI was reduced more than $50 \%$ or the lesion was completely absorbed compared with the initial CT scan. All 14 patients underwent the last CT before discharge, which showed more than $50 \%$ POI reduction rate of the lesions, and even the infected lesions in 6 patients were completely absorbed. Meanwhile, the body temperature remained normal for more than 3 days. There were no obvious respiratory symptoms, and the nucleic acid results were negative. Then, they were allowed to be discharged and isolated at home, and kept under health observation for 14 days. None of the 14 discharged cases had a subsequent positive nucleic acid test or manifested progression of pneumonia.

\section{Discussion}

CT imaging has become an effective tool for screening COVID-19 patients and assessing the severity of COVID-19 infection [17-22]. Considering that there is still no effective medicine to treat COVID-19, clinicians use different degrees of supportive therapy to intervene disease and then to see how disease progresses. To evaluate the therapeutic response in confirmed COVID-19 inpatients, follow-up CT examinations are required every 3 to 5 days. However, radiologists and clinicians still do not have a 
computerized tool to accurately quantify the treatment response. Deep learning (DL) has become a popular method in medical image analysis [23, 24], and it has been used to screen for and diagnose COVID-19 on CT images [15, 25, 26]. Li et al. [25] used a DL model to train a large chest CT dataset; their results showed sensitivity of $90 \%$, specificity of $96 \%$, and accuracy of 0.96 in identifying COVID-19. The accurate segmentation by the DL system is a basis for quantitative assessment of CT images $[15,27,28]$, which is necessary to track the progression of the disease and analyze longitudinal changes of COVID-19 throughout the treatment period $[29,30]$. In this study, we included the data from 14 patients with mildtype COVID-19 and evaluated longitudinally and quantitatively the changes on chest CT during COVID-19 by using a tool of a DL-based network (VB-Net). We believe that this DL-based CT system for COVID-19 quantification can facilitate clinical decisions on treatment.

VB-Net system is an application of image processing used for segmentation of the lung, lung lobes, and lung infection [15]. It can provide accurate quantitative data for medical research, including quantitative assessment of disease progression at follow-up, comprehensive evaluation of severity, visualization and quantification of the lesion distribution using percentage of infection (POI). Shi et al. [31] used the VB-Net system to compute chest CT images of 2685 patients, and showed sensitivity of $90.7 \%$, specificity of $83.3 \%$, and accuracy of $87.9 \%$. We used the VB-Net system to quantify longitudinal changes between the initial and follow-up CT scans of COVID-19 patients. Figure 2 shows a case of a 57-year-old COVID-19 patient with five follow-up CT scans. The changes in infection volume as well as GGOs and consolidation components were clearly visualized by using the infection region segmentation method and surface rendering technique.

Dynamic radiological features on chest CT images of COVID-19 have already been reported [32]. In this study, we found that the distribution of lesions at the initial CT scan was predominantly in the bilateral lower lobes in 14 patients. GGOs accounted for the largest volume of the lesions, and some lesions showed consolidation changes and fibrosis, consistent with the results reported in previous studies [3338]. Early fibrosis may correlate with good prognosis [39]. Six patients showed an increase in consolidation component at the first follow-up, and two of them showed progression as reflected in increased infected area of multiple lobes and denser consolidation components. Finally, consolidation components were gradually absorbed. We also showed that the components in the lesions could be identified by CT number. Namely, for all lesions, the VB-Net system can calculate the POI with a CT number in the predefined range. In this study, we found the highest POI for the CT number in the range $<-300 \mathrm{HU}$, indicating that GGOs were major components of the lesions. These chest CT radiological features were consistent with COVID-19. The overall POls of 14 patients showed that the range of CT number gradually decreased. These results indicate that the CT features of COVID-19 infected lesions can be visually displayed by using the DL-based CT system, which might help clinicians to manage patients with COVID-19.

Shan et al. [15] indicated that the POI estimated from CT scans correlated with the severity of pneumonia. With the VB-Net system, the POIs of the whole lung and lung lobes can be automatically calculated, and then, the severity of COVID-19 infection in the whole lung and each lobe can be quantified. Table 2 shows 
that the POI of the right lower lobe was higher than that of the other lobes, which is in agreement with the findings reported in previous studies [36-38]. In addition, the research works of DL also can be helpful in predicting COVID-19 disease progression. Cao et al. [29] have reported that the use of voxel-level DLbased CT segmentation of pulmonary opacities can improve their quantification and assess longitudinal progression of COVID-19. Huang et al. [30] collected CT images from 126 patients and calculated the percentage of GGOs in chest CT images; they found that the quantification of the lung infection could be further used for monitoring of the progression of COVID-19. In our study, the whole lung and lesions were segmented by VB-Net system, and the POls were calculated from follow-up CT scans of 14 COVID-19 patients. Our results suggested that the POls of most patients showed a gradual downward trend after antiviral and supportive treatment (Figure 1). These follow-up results suggest that clinically relevant supportive treatment can be reflected in changes in POIs. Therefore, our study illustrates the potential of DL-based CT system to provide objective quantitative assessment of pulmonary infection as well as of response to treatment in patients with COVID-19.

In summary, DL plays an important role in the delineation of infected lesions and quantification of COVID19 applications. It helps radiologists in accurately identifying lung infection and prompting quantitative analysis and diagnosis of COVID-19.

In addition, the discharge criteria of common COVID-19 patients were managed well by DL; however, six cases in our study showed "re-positive" results during the follow-up nucleic acid tests. The quantitative CT follow-up results showed that the absorption of the infected lesions gradually decreased. Currently, there are research reports on COVID-19 "re-positive" patients without worsening symptoms and chest CT findings. Additionally, the patients were isolated at the infection ward of the hospital and were not exposed to other confirmed or suspected patients, which indicated that these "re-positive" tests were not the result of a re-infection. After obtaining the "re-positive" results, in the six patients, the samples from multiple sites, including the nasopharynx, throat, and anal swabs, were tested for COVID-19 nucleic acid for more than 3 days, but all the test results were negative. The quantitative results of $C T$ before discharge showed that the reduction rate of $\mathrm{POI}$ in the lung lesions was more than $50 \%$, and even the infected lesions in those six patients with re-positive tests were completely absorbed. After discharge, all the patients underwent 14 days of isolation and health surveillance at home.

To ensure that patients are completely cured, analysis of IgM and IgG COVID-19-specific antibodies should be carried out for all discharged patients. Additionally, the CT scans of "re-positive" patients still showed abnormal lesions in the lungs at the follow-up visit, suggesting that more rigorous criteria are needed to evaluate the CT results so as to reduce the possibility of "re-positive" test results.

This study had several limitations. First, although DL has become an effective method in fighting against COVID-19, the CT data in COVID-19 patients may be incomplete and inaccurate, which creates difficulty in training an accurate segmentation and diagnostic network. Meanwhile, our current Al study for quantification of the lesions was based on a small sample, which may have led to overfitting of the results. Multicenter prospective studies with larger samples need to be conducted to further verify the 
conclusions of the present study. Additionally, our study did not analyze the COVID-19-specific IgG-and $\lg \mathrm{M}$ antibodies due to the unavailability of such data. Finally, more datasets should be established to include clinically collected CT images from patients with COVID-19.

\section{Conclusion}

The pandemic of COVID-19 continues. Every day, hundreds of thousands of new patients are diagnosed, which not only increases the diagnostic pressure on radiologists but also restricts the treatment capabilities of hospitals and intensive care units. The DL-based CT model can help radiologists to assess a rising number of examinations as well as help clinicians to acquire results much faster.

CT quantitative analysis might be an effective and important method for assessing the severity of COVID19 , which could guide clinicians to rapidly apply the appropriate treatment. Meanwhile, the objective evaluation of the course of the infection gives the possibility to choose the correct treatment, which could guide clinical decisions during management of patients with COVID-19. Additionally, CT characteristics and quantitative analysis based on Al should be evaluated strictly, and the discharge standard should be specified depending on the baseline, disease status, and other patient-related factors.

The follow-up of COVID-19 remains a challenge. We believe that CT-based quantitative assessment using DL has great potential to facilitate both the diagnosis and tracking of the management of COVID-19 patients.

\section{Abbreviations}

COVID-19, Coronavirus Disease 2019; RT-PCR, Reverse-transcriptase polymerase chain reaction; CT, Computed tomography; Al, Artificial intelligence; DL, Deep learning; IRB, Institutional review board; CDC, the Centers for Disease Control; POI, Percentage of infection; HU, Hounsfield units; ROI, Regions of interest; GGO, Ground glass opacities; SD, Standard deviation; IQR, Interquartile range

\section{Declarations}

\section{Acknowledgments}

The authors thank LetPub (www.letpub.com) for its linguistic assistance and scientific consultation during the preparation of this manuscript. The authors are grateful to Liang Mao and his team members from the United Imaging Intelligence Company (Shanghai, China) for the software and technical support.

\section{Authors' contributions}

All authors revised the manuscript critically for intellectual content and approved the final version for publication. JL: substantial contribution to conception and design of the study, and preparation and drafting of the article. WZ: substantial contribution to acquisition of data and input of raw data and 
validation of data. QL: substantial contribution to analysis and interpretation of data, including assessment of CT scans and quantification for COVID-19. GJ: substantial contribution to conception and design, with oversight and leadership responsibility for the research. Substantial contribution to revision of the manuscript. JY: substantial contribution to conception and design throughout evolution and realization of the study idea, and management and coordination responsibility for the research activity planning and execution. LL: substantial contribution to conception and design throughout evolution of overarching research goals and aims, management and coordination responsibility for the research activity planning and execution, and final approval of the manuscript. All authors read and approved the final manuscript.

\section{Funding}

This research was supported by the Guangdong Second Provincial General Hospital Youth Research Project (grant number, YQ2018-007).

\section{Availability of data and materials}

The datasets used and/or analyzed in this study are available from the corresponding author on reasonable request.

\section{Ethics approval and consent to participate}

Ethics Committee of Guangdong Second Provincial General Hospital approved this study.

\section{Consent for publication}

Consent for publication has been obtained.

\section{Competing interests}

The authors declare that they have no known competing financial interests or personal relationships that could have appeared to influence the work reported in this paper.

\section{References}

1. Zhu N, Zhang D, Wang W, Li X, Yang B, Song J, Zhao X, Huang B, Shi W, Lu R, et al: A Novel Coronavirus from Patients with Pneumonia in China, 2019.N Engl J Med 2020, 382:727-733. doi: 10.1056/NEJMoa2001017.

2. Huang C, Wang Y, Li X, Ren L, Zhao J, Hu Y, Zhang L, Fan G, Xu J, Gu X, et al: Clinical features of patients infected with 2019 novel coronavirus in Wuhan, China.Lancet 2020, 395:497-506. doi: 10.1016/S0140-6736(20)30183-5.

3. Novel Coronavirus (2019-nCoV) Situation Report-22. WHO Web site; [Accessed Feb 11, 2020]. https://www.who.int/docs/default-source/coronaviruse/situation-reports/20200211-sitrep-22- 
ncov.pdf?sfvrsn=fb6d49b1_2. Published Feb 11, 2020.

4. WHO. Coronavirus disease 2019 (COVID-19). Weekly Epidemiological Update - 23 February 2021. WHO Web site; [Accessed Feb 21, 2021]. https://www.who.int/publications/m/item/weeklyepidemiological-update-23-february-2021. Published Feb 23, 2021.

5. Ai T, Yang Z, Hou H, Zhan C, Chen C, Lv W, Tao Q, Sun Z, Xia L: Correlation of Chest CT and RT-PCR Testing in Coronavirus Disease 2019 (COVID-19) in China: A Report of 1014 Cases. Radiology 2020, 296:E32-E40. doi: 10.1148/radiol.2020200642.

6. Lei P, Fan B, Sun Y: COVID-19 Carrier or Pneumonia: Positive Real-Time Reverse-Transcriptase Polymerase Chain Reaction but Negative or Positive Chest CT Results. Korean J Radio/ 2020, 21:925928. doi: 10.3348/kjr.2020.0360.

7. Fang Y, Zhang H, Xie J, Lin M, Ying L, Pang P, Ji W: Sensitivity of Chest CT for COVID-19: Comparison to RT-PCR.Radiology 2020, 296:E115-E117. doi: 10.1148/radiol.2020200432.

8. Xiong Y, Sun D, Liu Y, Fan Y, Zhao L, Li X, Zhu W: Clinical and High-Resolution CT Features of the COVID-19 Infection: Comparison of the Initial and Fol low-up Changes.Invest Radio/2020, 55(6):332339. doi: 10.1097/RLI.0000000000000674.

9. Liu Z, Jin C, Wu CC, Liang T, Zhao H, Wang Y, Wang Z, Li F, Zhou J, Cai S, et al: Association between Initial Chest CT or Clinical Features and Clinical Course in Patients with Coronavirus Disease 2019 Pneumonia.Korean J Radiol 2020:21(26):736-745. doi: 10.3348/kj.2020.0171.

10. Jin S, Wang B, Xu H, Luo C, Wei L, Zhao W, Hou X, Ma W, Xu Z, Zheng Z, et al: Al-assisted CT imaging analysis for COVID-19 screening: Building and deploying a medical Al system in four weeks.medRxiv 2020:03.19.20039354. https://doi.org/10.1101/2020.03.19.20039354.

11. Kermany DS, Goldbaum M, Cai W, Valentim CCS, Liang H, Baxter SL, McKeown A, Yang G, Wu X, Yan F, et al: Identifying Medical Diagnoses and Treatable Diseases by Image-Based Deep Learning.Cell 2018, 172:1122-1131.e1129. doi: 10.1148/radiol.2020200241.

12. Anthimopoulos M, Christodoulidis S, Ebner L, Christe A, Mougiakakou S: Lung Pattern Classification for Interstitial Lung Diseases Using a Deep Convolutional Neural Network.IEEE Trans Med Imaging 2016, 35:1207-1216. doi: 10.1109/TMI.2016.2535865.

13. Joseph Bullock AL, Katherine Hoffmann Pham, Cynthia Sin Nga Lam, Miguel Luengo-Oroz: Mapping the Landscape of Artificial Intelligence Applications against COVID-19.arXiv 2020:2003.11336. doi: arXiv:2003.11336.

14. The China Guidelines for the Diagnosis and Treatment plan of Coronavirus Disease 2019 (COVID-19) (Trial Version 7). Web site [Accessed Mar 3, 2020]. http://www.nhc.gov.cn/yzygj/s7652m/202003/a31191442e29474b98bfed5579d5af95.shtml

15. Fei Shan YG, Jun Wang, Weiya Shi, Nannan Shi, Miaofei Han, Zhong Xue, Dinggang Shen, Yuxin Shi: Lung Infection Quantification of COVID-19 in CT Images with Deep Learning.arXiv 2020:2003.04655. doi: arXiv:2003.04655.

16. Milletari F NN, Ahmadi S-A: V-net: Fully convolutional neural networks for volumetric medical image segmentation. 2016 Fourth International Conference on 3D Vision (3DV). IEEE 2016, p:565-571. doi: 
10.1109/3DV.2016.79.

17. Kanne JP: Chest CT Findings in 2019 Novel Coronavirus (2019-nCoV) Infections from Wuhan, China: Key Points for the Radiologist.Radiology 2020, 295:16-17. doi: 10.1148/radiol.2020200241.

18. Bernheim A, Mei X, Huang M, Yang Y, Fayad ZA, Zhang N, Diao K, Lin B, Zhu X, Li K, et al: Chest CT Findings in Coronavirus Disease-19 (COVID-19): Relationship to Duration of Infection.Radiology 2020, 295:200463. doi: 10.1148/radiol.2020200463.

19. Zokaeinikoo M, Kazemian P, Mitra P, Kumara S: AIDCOV: An Interpretable Artificial Intelligence Model for Detection of COVID-19 from Chest Radiography Images.medRxiv 2020:2020.2005.2024.20111922. https://doi.org/10.1101/2020.05.24.20111922.

20. Sun D, Li X, Guo D, Wu L, Chen T, Fang Z, Chen L, Zeng W, Yang R: CT Quantitative Analysis and Its Relationship with Clinical Features for Assessing the Severity of Patients with COVID-19.Korean J Radiol 2020, 21:859-868. doi: 10.3348/kjr.2020.0293.

21. Yin X, Min X, Nan Y, Feng Z, Li B, Cai W, Xi X, Wang L: Assessment of the Severity of Coronavirus Disease: Quantitative Computed Tomography Parameters versus Semiquantitative Visual Score.Korean J Radiol 2020, 21:998-1006. doi: 10.3348/kjr.2020.0423.

22. Zhao X, Liu B, Yu Y, Wang X, Du Y, Gu J, Wu X: The characteristics and clinical value of chest CT images of novel coronavirus pneumonia.Clin Radiol 2020, 75:335-340. doi:

10.1016/j.crad.2020.03.002.

23. Pang T, Guo S, Zhang X, Zhao L: Automatic Lung Segmentation Based on Texture and Deep Features of HRCT Images with Interstitial Lung Disease.Biomed Res Int 2019, 7:1-8. doi:

10.1155/2019/2045432.

24. Park B, Park H, Lee SM, Seo JB, Kim N: Lung Segmentation on HRCT and Volumetric CT for Diffuse Interstitial Lung Disease Using Deep Convolutional Neural Networks.J Digit Imaging 2019, 32:10191026. doi: 10.1007/s10278-019-00254-8.

25. Li Z, Zhong Z, Li Y, Zhang T, Gao L, Jin D, Sun Y, Ye X, Yu L, Hu Z, et al: From community-acquired pneumonia to COVID-19: a deep learning-based method for quantitative analysis of COVID-19 on thick-section CT scans.Eur Radiol 2020; 30(12):6828-6837. doi: 10.1007/s00330-020-07042-x.

26. Shi F, Wang J, Shi J, Wu Z, Wang Q, Tang Z, He K, Shi Y, Shen D: Review of Artificial Intelligence Techniques in Imaging Data Acquisition, Segmentation and Diagnosis for COVID-19.IEEE Rev Biomed Eng 2020. doi: 10.1109/RBME.2020.2987975.

27. Tang L, Zhang X, Wang Y, Zeng X: Severe COVID-19 Pneumonia: Assessing Inflammation Burden with Volume-rendered Chest CT.Radiology: Cardiothoracic Imaging 2020, 2:e200044. https://doi.org/10.1148/ryct.2020200044.

28. Shen C, Yu N, Cai S, Zhou J, Sheng J, Liu K, Zhou H, Guo Y, Niu G: Quantitative computed tomography analysis for stratifying the severity of Coronavirus Disease 2019.J Pharm Anal 2020, 10:123-129. doi: 10.1016/j.jpha.2020.03.004.

29. Cao Y, Xu Z, Feng J, Jin C, Han X, Wu H, Shi H: Longitudinal Assessment of COVID-19 Using a Deep Learning-based Quantitative CT Pipeline: Illustration of Two Cases.Radiology: Cardiothoracic 
Imaging 2020, 2:e200082. https://doi.org/10.1148/ryct.2020200082.

30. Huang L, Han R, Ai T, Yu P, Kang H, Tao Q, Xia L: Serial Quantitative Chest CT Assessment of COVID19: Deep-Learning Approach.Radiology: Cardiothoracic Imaging 2020, 2:e200075. https://doi.org/10.1148/ryct.2020200075.

31. Feng Shi LX, Fei Shan, Dijia Wu, Ying Wei, Huan Yuan, Huiting Jiang, Yaozong Gao, He Sui, Dinggang Shen: Large-Scale Screening of COVID-19 from Community Acquired Pneumonia using Infection Size-Aware Classification.arXiv 2020:2003.09860. doi: arXiv:2003.09860.

32. Pan F, Ye T, Sun P, Gui S, Liang B, Li L, Zheng D, Wang J, Hesketh RL, Yang L, Zheng C: Time Course of Lung Changes On Chest CT During Recovery From 2019 Novel Coronavirus (COVID-19).Radiology 2020, 295:715-721. doi: 10.1148/radiol.2020200370.

33. Caruso D, Zerunian M, Polici M, Pucciarelli F, Polidori T, Rucci C, Guido G, Bracci B, de Dominicis C, Laghi A: Chest CT Features of COVID-19 in Rome, Italy.Radiology 2020, 296:E79-E85. doi: 10.1148/radiol.2020201237.

34. Carotti M, Salaffi F, Sarzi-Puttini P, Agostini A, Borgheresi A, Minorati D, Galli M, Marotto D, Giovagnoni $A$ : Chest CT features of coronavirus disease 2019 (COVID-19) pneumonia: key points for radiologists.Radiol Med 2020, 125:636-646. doi: 10.1007/s11547-020-01237-4.

35. Ye Z, Zhang Y, Wang Y, Huang Z, Song B: Chest CT manifestations of new coronavirus disease 2019 (COVID-19): a pictorial review.Eur Radiol 2020, 30:4381-4389. doi: 10.1007/s00330-020-06801-0.

36. Colombi D, Bodini FC, Petrini M, Maffi G, Morelli N, Milanese G, Silva M, Sverzellati N, Michieletti E: Well-aerated Lung on Admitting Chest CT to Predict Adverse Outcome in COVID-19

Pneumonia.Radiology 2020, 296:E86-E96. doi: 10.1148/radiol.2020201433.

37. Chung M, Bernheim A, Mei X, Zhang N, Huang M, Zeng X, Cui J, Xu W, Yang Y, Fayad ZA, et al: CT Imaging Features of 2019 Novel Coronavirus (2019-nCoV).Radiology 2020, 295:202-207. doi: 10.1148/radiol.2020200230.

38. Song F, Shi N, Shan F, Zhang Z, Shen J, Lu H, Ling Y, Jiang Y, Shi Y: Emerging 2019 Novel Coronavirus (2019-nCoV) Pneumonia.Radiology 2020, 295:210-217. doi:

10.1148/radiol.2020200274.

\section{Tables}

Table 1. Demographic and Clinical Characteristics of Patients with COVID-19 $(n=14)$ 


\begin{tabular}{|ll|}
\hline Variables & Number (\%) \\
\hline Age (years) & $53 \pm 14$ \\
\hline Sex (male/female), $\mathrm{n}(\%)$ & $6(42.9)$ / $8(57.1)$ \\
\hline Exposure history & \\
\hline Lived in Hebei Province, China, $\mathrm{n}(\%)$ & $10(71.4)$ \\
\hline Exposure to infected cases, $\mathrm{n}(\%)$ & $4(28.6)$ \\
\hline Initial symptoms & \\
\hline Fever, $\mathrm{n}(\%)$ & $12(85.7)$ \\
\hline Cough, $\mathrm{n}(\%)$ & $5(35.7)$ \\
\hline Sore throat, $\mathrm{n}(\%)$ & $2(14.3)$ \\
\hline Laboratory findings & \\
\hline Reduced lymphocyte count, $\mathrm{n}(\%)$ & $11(78.6)$ \\
\hline Reduced leukocyte count, $\mathrm{n}(\%)$ & $5(35.7)$ \\
\hline Reduced CD ${ }^{+}$T lymphocyte count, $\mathrm{n}(\%)$ & $12(85.7)$ \\
\hline Reduced CD4 ${ }^{+}$T lymphocyte count, $\mathrm{n}(\%)$ & $12(85.7)$ \\
\hline Reduced CD ${ }^{+}$T lymphocyte count, $\mathrm{n}(\%)$ & $8(57.1)$ \\
\hline Elevated C-reactive protein, $\mathrm{n}(\%)$ & $10(71.4)$ \\
\hline Comorbidity & $3(21.4)$ \\
\hline Hyperuricemia, $\mathrm{n}(\%)$ & $2(8.7)$ \\
\hline Hypertension, $\mathrm{n}(\%)$ & \\
\hline Chronic hepatitis B, $\mathrm{n}(\%)$ & $(4.3)$ \\
\hline Chronic obstructive pulmonary disease, $\mathrm{n}(\%)$ & \\
\hline
\end{tabular}

Table 2. Percentage of Infection (POI) at Initial and Follow-up CT Images Based on VB-Net System in Patients with COVID-19 ( $n=14)$ 


\begin{tabular}{|c|c|c|c|c|c|c|}
\hline \multirow[t]{2}{*}{ Accuracy metrics } & Initial & $\begin{array}{l}1 \text { st follow- } \\
\text { up }\end{array}$ & $\begin{array}{l}\text { 2nd follow- } \\
\text { up }\end{array}$ & $\begin{array}{l}\text { 3rd follow- } \\
\text { up }\end{array}$ & $\begin{array}{l}\text { 4th follow- } \\
\text { up }\end{array}$ & \multirow[t]{2}{*}{$p^{\star}$} \\
\hline & POI (\%) & POI (\%) & POI (\%) & POI (\%) & POI (\%) & \\
\hline Whole lung & $\begin{array}{l}5.0(0.8- \\
9.8)\end{array}$ & $\begin{array}{l}3.8(0.4- \\
11.0)\end{array}$ & $\begin{array}{l}2.0(0.1- \\
8.3)\end{array}$ & $\begin{array}{l}2.0(0.0- \\
6.4)\end{array}$ & $\begin{array}{l}0.2(0.0- \\
2.1)\end{array}$ & $\dot{0.001}$ \\
\hline Left lung & $\begin{array}{l}0.9(0.2- \\
9.3)\end{array}$ & $\begin{array}{l}0.8(0.2- \\
10.7)\end{array}$ & $\begin{array}{l}0.8(0.1- \\
7.2)\end{array}$ & $\begin{array}{l}0.3(0.0- \\
4.0)\end{array}$ & $\begin{array}{l}0.1(0.0- \\
1.0)\end{array}$ & $\begin{array}{l}<.001 \\
0.00\end{array}$ \\
\hline Left upper lobe & $\begin{array}{l}0.3(0.0- \\
2.6)\end{array}$ & $\begin{array}{l}0.1(0.0- \\
3.1)\end{array}$ & $\begin{array}{l}0.1(0.0- \\
2.9)^{(0.0}\end{array}$ & $\begin{array}{l}0.1(0.0- \\
0.9)\end{array}$ & $\begin{array}{l}0.0(0.0- \\
0.2)\end{array}$ & $\stackrel{<}{0.001}$ \\
\hline Left lower lobe & $\begin{array}{l}0.6(0.1- \\
7.2)\end{array}$ & $\begin{array}{l}0.8(0.1- \\
8.7)\end{array}$ & $\begin{array}{l}0.8(0.0- \\
4.6)\end{array}$ & $\begin{array}{l}0.3(0.0- \\
1.9)\end{array}$ & $\begin{array}{l}0.0(0.0- \\
0.4)\end{array}$ & 0.001 \\
\hline Right lung & $\begin{array}{l}6.8(1.1- \\
12.2)\end{array}$ & $\begin{array}{l}5.7(0.6- \\
12.3)\end{array}$ & $\begin{array}{l}2.7(0.0- \\
10.4)\end{array}$ & $\begin{array}{l}2.5(0.0- \\
7.8)\end{array}$ & $\begin{array}{l}0.7(0.0- \\
1.5)\end{array}$ & $\dot{0} .001$ \\
\hline Right upper lobe & $\begin{array}{l}0.5(0.1- \\
2.8)\end{array}$ & $\begin{array}{l}0.2(0.0- \\
2.4)\end{array}$ & $\begin{array}{l}0.1(0.0- \\
1.9)^{(0-}\end{array}$ & $\begin{array}{l}0.0(0.0- \\
1.8)\end{array}$ & $\begin{array}{l}0.0(0.0- \\
0.1)\end{array}$ & 0.001 \\
\hline Right middle lobe & $\begin{array}{l}0.1(0.0- \\
1.2)\end{array}$ & $\begin{array}{l}0.1(0.0- \\
1.5)\end{array}$ & $\begin{array}{l}0.1(0.0- \\
1.0)\end{array}$ & $\begin{array}{l}0.0(0.0- \\
0.4)\end{array}$ & $\begin{array}{l}0.0(0.0- \\
0.1)\end{array}$ & $\begin{array}{l}<.001 \\
0.00\end{array}$ \\
\hline Right lower lobe & $\begin{array}{l}6.5(0.9- \\
9.0)\end{array}$ & $\begin{array}{l}4.4(0.5- \\
9.8)\end{array}$ & $\begin{array}{l}2.4(0.0- \\
8.3)\end{array}$ & $\begin{array}{l}2.7(0.0- \\
5.7)\end{array}$ & $\begin{array}{l}0.6(0.0- \\
1.1)\end{array}$ & $\stackrel{<}{0.001}$ \\
\hline \multicolumn{7}{|l|}{$\begin{array}{l}\text { Range of CT } \\
\text { number }\end{array}$} \\
\hline$<-300 \mathrm{HU}$ & $\begin{array}{l}4.2(0.7- \\
8.8)\end{array}$ & $\begin{array}{l}3.7(0.3- \\
9.4)\end{array}$ & $\begin{array}{l}1.8(0.1- \\
7.3)\end{array}$ & $\begin{array}{l}1.9(0.0- \\
6.1)\end{array}$ & $\begin{array}{l}0.4(0.0- \\
1.2)\end{array}$ & $\dot{0.001}$ \\
\hline $\begin{array}{l}-300 \mathrm{HU} \text { to } 49 \\
\mathrm{HU}\end{array}$ & $\begin{array}{l}0.6(0.1- \\
1.4)\end{array}$ & $\begin{array}{l}0.2(0.0- \\
2.1)\end{array}$ & $\begin{array}{l}0.1(0.0- \\
2.0)\end{array}$ & $\begin{array}{l}0.1(0.0- \\
0.3)\end{array}$ & $\begin{array}{l}0.0(0.0- \\
0.1)\end{array}$ & $\dot{0} .001$ \\
\hline$\geq 50 \mathrm{HU}$ & $\begin{array}{l}0.0(0.0- \\
0.1)\end{array}$ & $\begin{array}{l}0.0(0.0- \\
0.1)\end{array}$ & $\begin{array}{l}0.0(0.0- \\
0.1)\end{array}$ & 0 & 0 & 0.036 \\
\hline
\end{tabular}

$\mathrm{POI}$ values are presented as median and interquartile range (IQR, the first to the third quartile). ${ }^{*} p$ based on the Friedman test. $\mathrm{POI}=$ percentage of infection .

\section{Figures}




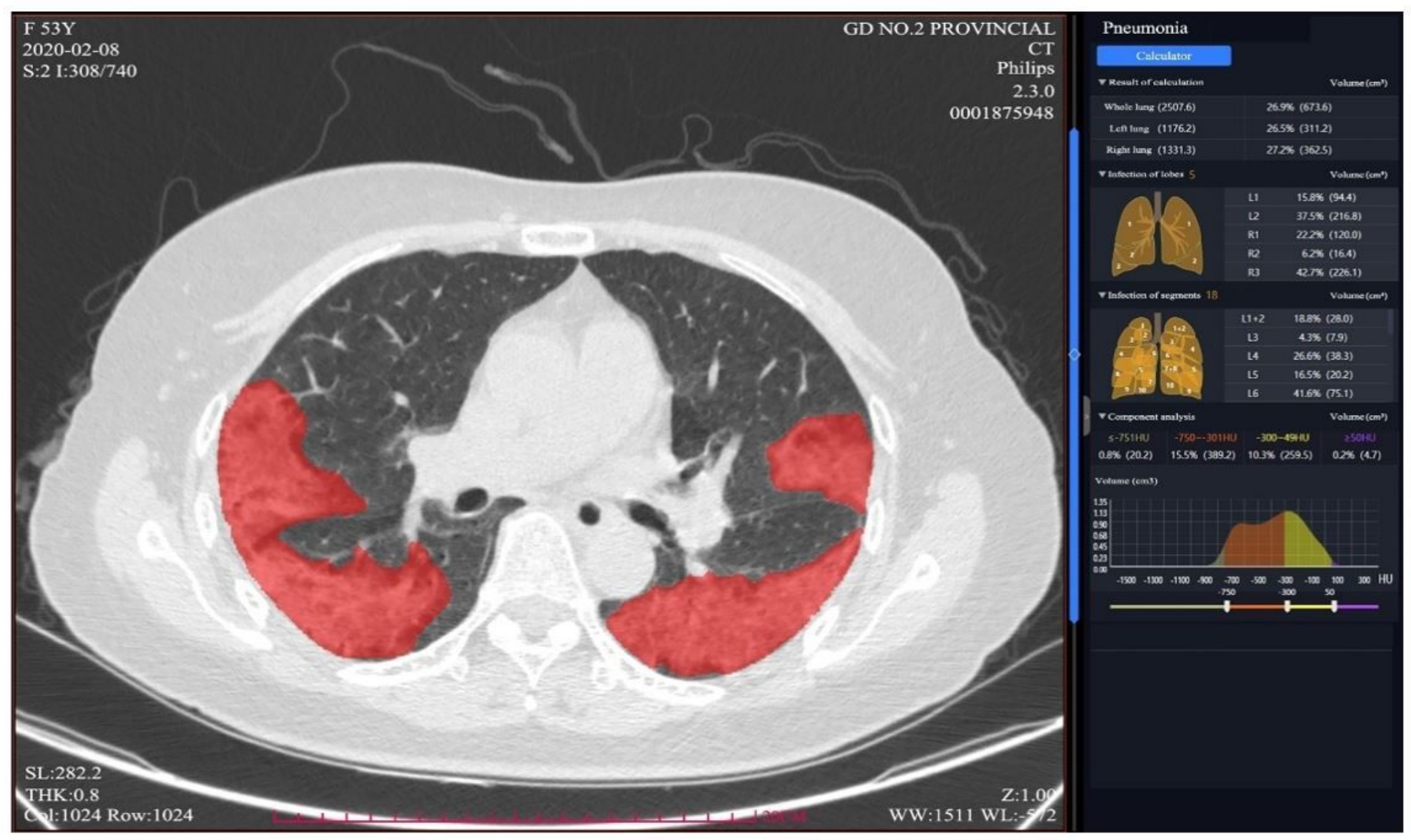

Figure 1

Working model of the VB-Net system in chest CT of a 53-year-old woman. The left panel shows the results predicted by the VB-Net system. The results of calculation are shown in the right panel. The POls of the total lung, lobes, and segments are shown in the upper and middle right panels. The volume of different components within the infected region is shown in the lower right panel. CT = computed tomography, $\mathrm{POI}=$ percentage of infection 


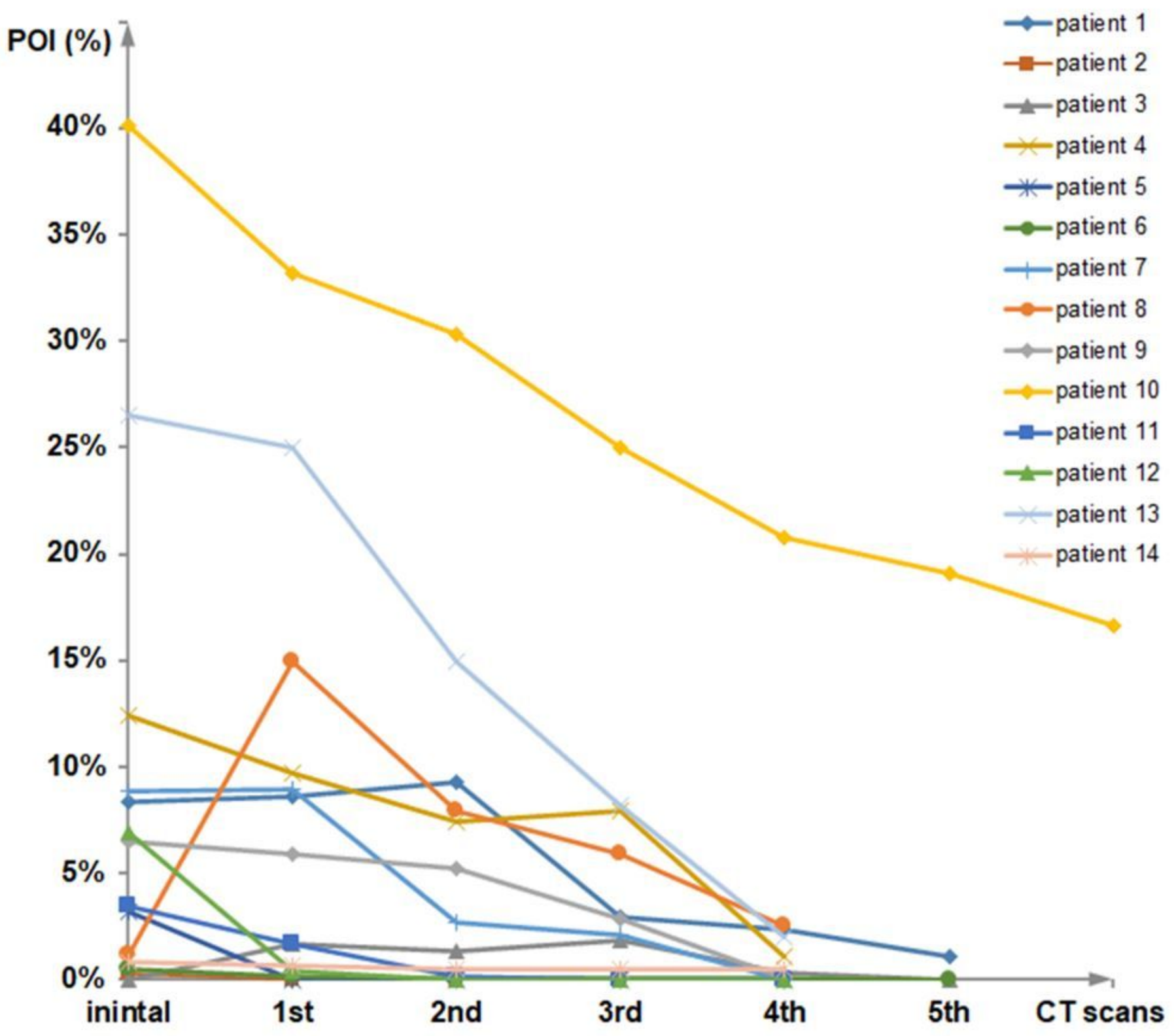

Figure 2

Quantitative results of follow-up CT scans in 14 patients. The changes in POI values show the progression and gradual recovery in the $\mathrm{CT}$ scans. $\mathrm{POI}=$ percentage of infection, $\mathrm{CT}=$ computed tomography 


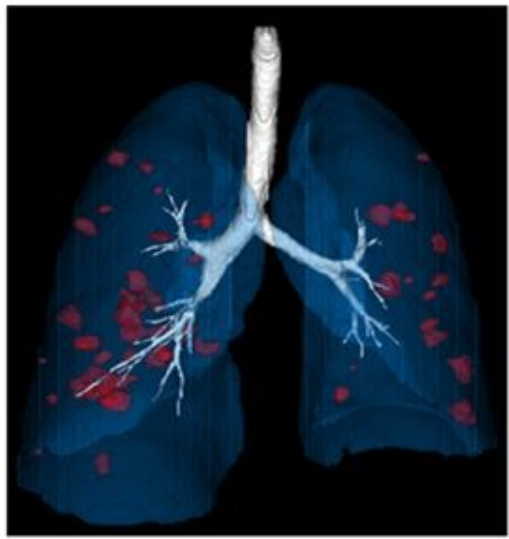

A

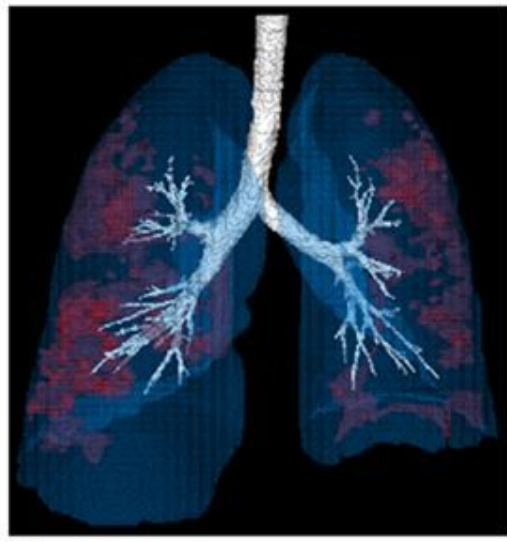

D

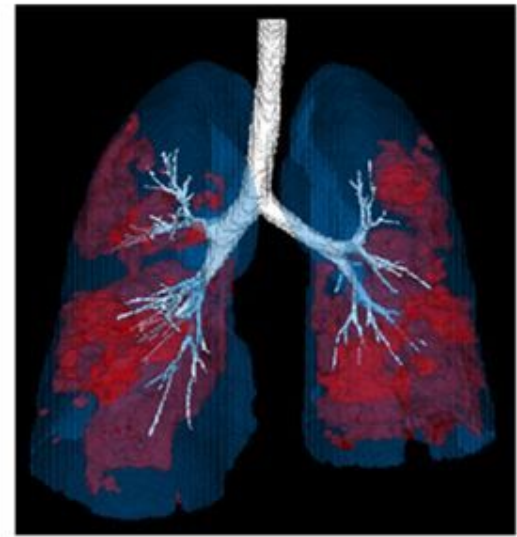

B

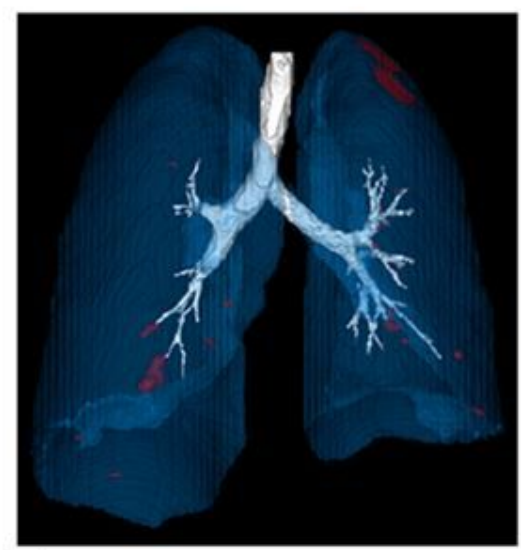

E

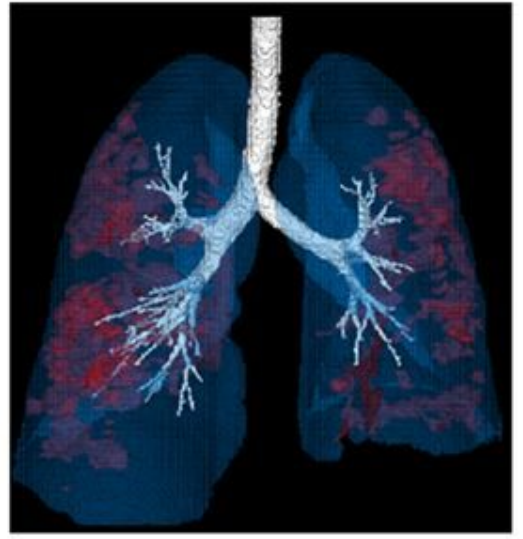

C

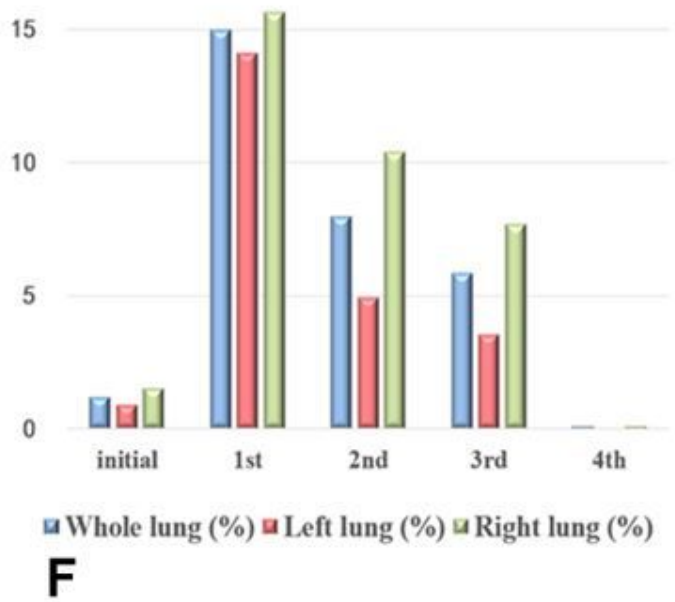

\section{Figure 3}

Initial and follow-up results in a 57-year-old man with confirmed mild-type COVID-19. Red color indicates infected lesions inside the whole lung $(A-C)$. A. February 1, 2020. $P O I=1.2 \%, B$. February 6, 2020. $P O I=$ $15.0 \%$, C. February $14,2020 . \mathrm{POI}=8.0 \%$, D. February $18,2020 . \mathrm{POI}=5.9 \%$, E. March 8, 2020. POI $=0.1 \%$. $\mathrm{F}$. The $\mathrm{POI}$ values first show the progression and then a gradual improvement from February 1 to March 8 , 2020. $\mathrm{POI}=$ percentage of infection 